\title{
CATIONIC PHOTOCURED EPOXY NANOCOMPOSITES FILLED WITH DIFFERENT CARBON FILLERS
}

\author{
M. Martin-Gallego ${ }^{1 *}$, M. Hernández ${ }^{1}$, V. Lorenzo ${ }^{2}$, R. Verdejo ${ }^{1}$, M.A. Lopez- \\ Manchado $^{1}$, M. Sangermano ${ }^{3 *}$
}

${ }^{1}$ Instituto de Ciencia y Tecnologia de Polimeros, ICTP-CSIC, Juan de la Cierva, 3 28006-Madrid, Spain.

${ }^{2}$ Univ Politecnica Madrid, ETS Ingenieros Industriales, Jose Gutierrez Abascal, 2 28006 Madrid, Spain.

${ }^{3}$ Politecnico di Torino, Dipartimento di Scienza Applicata e Tecnologia, C.so Duca degli Abruzzi 24, 10129, Torino.

\begin{abstract}
In this work, the effect of several carbon fillers, exfoliated graphite (EG), functionalized graphene sheets (FGS), multi-walled carbon nanotubes (MWCNTs), and oxidized multi-walled carbon nanotubes (f-MWCNTs), were compared on the curing process and physical properties of a cationically photocurable epoxy resin. The extent of the photopolymerization was monitored by Real-Time FTIR spectroscopy. It was found that all the nanofillers induced a delaying of the curing reaction probably due to a shielding effect as well as to an increase of the viscosity by the nanofiller. All the systems showed an electrical percolation threshold, but with MWCNTs was attained at a lower concentration $(<0.1 \mathrm{wt} . \%)$. In addition, FGS were the most efficient filler for epoxy resins, deduced by the dynamic mechanical and microindentation measurements. An increase of more than $20^{\circ} \mathrm{C}$ in the glass transition temperature was observed with the addition of 1 wt. $\%$ of FGS.
\end{abstract}

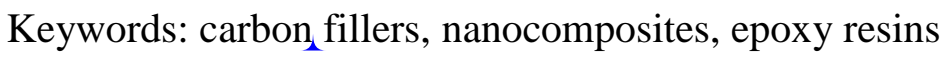


*Corresponding author: m.martingallego@ictp.csic.es or marco.sangermano@polito.it;

Tel: +34 912587424 or Tel: +390110904651

\section{INTRODUCTION}

Polymer nanocomposite coatings are attracting great attention in recent decades due to the possibility of adding valuable properties to the matrix. This multifunctionality is essential since polymer coatings should not only meet decorative or protective functions, they should also provide other requirements like electrostatic discharge, high mechanical performance, large operating temperature range and chemical resistance among others [1]. One approach to reach these features is the addition of nanofillers with remarkable mechanical, electrical and thermal properties. In this sense, carbon based nanofiller like carbon nanotubes (CNTs), functionalized graphene sheets (FGS) and expanded graphite (EG) appear as promising candidates for the reinforcement of polymer matrices $[2,3]$.

Numerous reports have already demonstrated the positive effects of CNTs and graphite on epoxy resins [3,4]. Graphene is following the same trend in the last years [5], but just a few of them are related to UV-photocured nanocomposites [6, 7]. Nowadays, UVcuring processes are becoming a very reliable alternative to the thermal process due to their particular characteristics [8]: fast transformation of the liquid monomer into a thin solid crosslinked coating with taylored physical and chemical properties without the need of using a solvent, so they are considered as environmental friendly processes. This technique is usually performed at room temperature allowing us to save energy. Furthermore, the use of the cationic photopolymerization provides other advantages compared to the radical one [9]. 
In this work, we focus on the effect of several carbon based nanofiller on the curing reaction of a photocurable epoxy resin by evaluating the photopolymerization process in real time. We also analyzed the influence of the structural parameters of the carbon nanofillers, such as geometry, structure and chemical composition on the conductivity, dielectric permittivity, percolation threshold and reinforcing effect on epoxy nanocomposites.

\section{EXPERIMENTAL SECTION:}

\section{Materials}

The UV-curable epoxy resin was the bis-cycloaliphatic diepoxy resin 3,4epoxycyclohexylmethyl-3',4'-epoxycyclohexyl carboxylate (CE) and the triphenylsulfonium hexafluoroantimonate $\left(\mathrm{Ph}_{3} \mathrm{~S}^{+} \mathrm{SbF}_{6}^{-}\right)$was used as photoinitiator. Both products were purchased from Sigma-Aldrich and used as received without any further purification.

Multi-walled carbon nanotubes (MWCNTs) were synthesized by the chemical vapour deposition (CVD) injection method using a solution of $3 \mathrm{wt} . \%$ of ferrocene in toluene at $760{ }^{\circ} \mathrm{C}$ under inert atmosphere as previously reported [10]. They are characterized by a diameter around 40-60 $\mathrm{nm}$ and a length of approximately $100 \mu \mathrm{m}$. We employed these MWCNTs without any further purification. These MWCNTs were then acid treated (fMWCNTs) in reflux in a 3:1 concentrated sulfuric/nitric acid mixture at $120{ }^{\circ} \mathrm{C}$ for 30 min, filtered and washed until neutral pH. f-MWCNTs were dried overnight at $120{ }^{\circ} \mathrm{C}$. The acid treatment provides hydroxyl and carbonyl functional groups on the MWCNT surface $[10,11]$. 
Functionalized graphene sheets (FGS) were also synthesized in our laboratories by the reduction and thermal exfoliation of graphite oxide at $1000{ }^{\circ} \mathrm{C}$ for $30 \mathrm{~s}$. Graphite oxide was previously obtained using natural graphite flakes (purum powder $\leq 0.1 \mathrm{~mm}$, SigmaAldrich) following the Brödie method. Graphene produced through this method leads to the formation of single graphene layers or stacks of up to 7 sheets with hydroxyl, carbonyl and epoxy groups on their surface [12]. A full description of the synthesis and characterization of the FGS can be found elsewhere [13].

Expanded graphite was prepared using a mixture of graphite flakes (Sigma-Aldrich) with concentrated sulfuric acid and nitric acid (4:1, v/v) stirred at room temperature. This acid treated natural graphite was washed and dryed to remove any remaining solvent. The dried filler were treated at $1050{ }^{\circ} \mathrm{C}$ for $30 \mathrm{~s}$ to obtain expanded graphite filler [14].

\section{Sample preparation}

Carbon-based nanofillers were directly dispersed into the epoxy resin using an IKA Ultraturrax at $30000 \mathrm{rpm}$ for $10 \mathrm{~min}$. Then, the mixtures were placed in an ultrasonic bath for another $10 \mathrm{~min}$. Table 1 summarizes the different formulations analysed in this study.

Table 1:filler contents added to the epoxy resin.

\begin{tabular}{ccccc}
\hline & MWCNT & $f-M W C N T$ & $F G S$ & $E G$ \\
\hline wt.\% range & $0.1-1$ & $0.5-1$ & $0.5-1.5$ & $1-5$ \\
\hline
\end{tabular}


The photoinitiator was added just before UV-irradiation in a proportion of $2 \mathrm{wt} \%$ respect to the resin.

For dielectric tests, the formulations were coated on glass substrates and cured with UV-light. The UV-induced polymerization was promoted by UV irradiation (UV lamp Italyquartz), with a light intensity on the surface of the sample of about $55 \mathrm{~mW} / \mathrm{cm}^{2}$ during $15 \mathrm{~min}$ ( $7.5 \mathrm{~min}$ each side of the sample) while $3 \mathrm{~min}$ were applied to the transparent pristine epoxy resin. Homogeneous disc-shape nanocomposite coatings of about $150 \mu \mathrm{m}$ in thickness and $2 \mathrm{~cm}$ of diameter were produced. These discs were cut to prepare rectangular samples for the DMTA and surface hardness tests.

\section{Sample characterization}

The progress of the photopolymerization process was followed by real time FTIR in a Thermo-Nicolet 5700 spectroscopy. During RT-FTIR analyses, the spectra were obtained co-adding 7 individual scans at $8 \mathrm{~cm}^{-1}$ resolution. $25 \mu \mathrm{m}$-thickness films were coated on silicon wafers and simultaneously exposed to the UV-beam (Hamamatsu LC8 portable UV-lamp) with an intensity of $35 \mathrm{~mW} / \mathrm{cm}^{2}$ for $3 \mathrm{~min}$ to induce polymerization, and to the IR-beam, to make an in-situ evaluation of the extent of reaction. The conversion of the epoxy group was followed by monitoring the decrease in the absorbance of the epoxy groups centered at $750 \mathrm{~cm}^{-1}$ and normalized by the carbonyl peak centered at around $1700 \mathrm{~cm}^{-1}$. The conversion of the cured samples used for the dielectric and mechanical tests was determined by carrying out single spectrum before and after 5 minutes of irradiation with the UV lamp (Italyquartz) at a light intensity of $55 \mathrm{~mW} / \mathrm{cm}^{2}$ on a coated film of about $25 \mu \mathrm{m}$-thickness.

Gel content values of the cured coatings were obtained by measuring weight loss after $24 \mathrm{~h}$ in chloroform at room temperature (ASTM D2765-84). 
Broadband dielectric spectroscopy was performed on an ALPHA high-resolution dielectric analyser (Novocontrol Technologies GmbH, Hundsangen, Germany). The photocured films were held in the dielectric cell between two parallel gold-plated electrodes. The dielectric response of each sample was assessed by measuring the complex permittivity $\varepsilon^{*}(\omega)=\varepsilon^{\prime}(\omega)$ - je $\varepsilon^{\prime \prime}(\omega)$ over a frequency range window of $10^{1}$ to $10^{7}$ $\mathrm{Hz}$ at room temperature. The amplitude of the alternating electric current applied to the samples was $1 \mathrm{~V}$. The AC conductivity were measured in the same conditions as $\varepsilon^{*}$ and the results were given directly by the dielectric analyzer. In a conducting composite, the conductivity is composed of two terms: $\sigma(\omega)=\sigma_{\mathrm{DC}}+\mathrm{A} \omega^{\mathrm{x}}$ Where $\sigma_{\mathrm{DC}}$ is the direct current conductivity and $\mathrm{x}$ is an exponent which describes the frequency $(\omega)$ dependence of $\sigma(\omega)$. For insulating materials $x=1$. The term $\sigma_{\mathrm{DC}}$ appears as a plateau at low frequencies in the experiments and it was obtained extrapolating the broadband AC conductivity to $10^{-1} \mathrm{~Hz}$ when a plateau is reached.

Scanning electron microscopy (SEM) was carried out in a Philips XL30 ESEM at 25 $\mathrm{kV}$ to study the morphology and dispersion states of the cryo-fractured samples. The study of the dispersion FGS was carried out using transmission electron microscopy (TEM Leo 910 microscope at an acceleration voltage of $80 \mathrm{kV}$ ). Ultra-thin sections of the sample containing $1 \mathrm{wt} . \%$ of FGS were prepared by cryo-ultramicrotomy at $-140{ }^{\circ} \mathrm{C}$ (Leica EM UC6).

Dynamic mechanical analysis was performed on a Mettler Toledo $861^{\mathrm{e}}$ instrument at 1 $\mathrm{Hz}$ frecquency in tensile configuration. Loss factor $(\tan \delta)$ the elastic modulus (E') were measured from room temperature to $250^{\circ} \mathrm{C}$. 
Surface hardness tests were performed at room temperature with a standard microhardness tester Shimadzu model DUH211S. A load of $10 \mathrm{mN}$ with a loading speed of $1.46 \mathrm{mN} / \mathrm{s}$ was applied and held for $5 \mathrm{~s}$ before unloading.

\section{RESULTS AND DISCUSSION:}

\section{Photopolymerization Process}

The influence of the different carbon fillers on the cationic photopolymerization reaction of an epoxy resin was evaluated by real time FTIR, keeping the filler content constant at $1 \mathrm{wt} . \%$. Figure 1 shows the evolution of the spectra as function of irradiation time recorded for the pure epoxy resin. The decrease of the epoxy group band, centered around $750 \mathrm{~cm}^{-1}$, as a function of time provides an indication of the extent of the reaction. Figure 2 shows the conversion curves of all samples calculated from the normalized absorbance of the epoxy by the carbonyl band. From this plot, we can get some qualitative information; the slope of the initial linear segment of the curves is related to the photopolymerization rate and the final value to the epoxy group conversion reached at the end of the UV-curing process. 


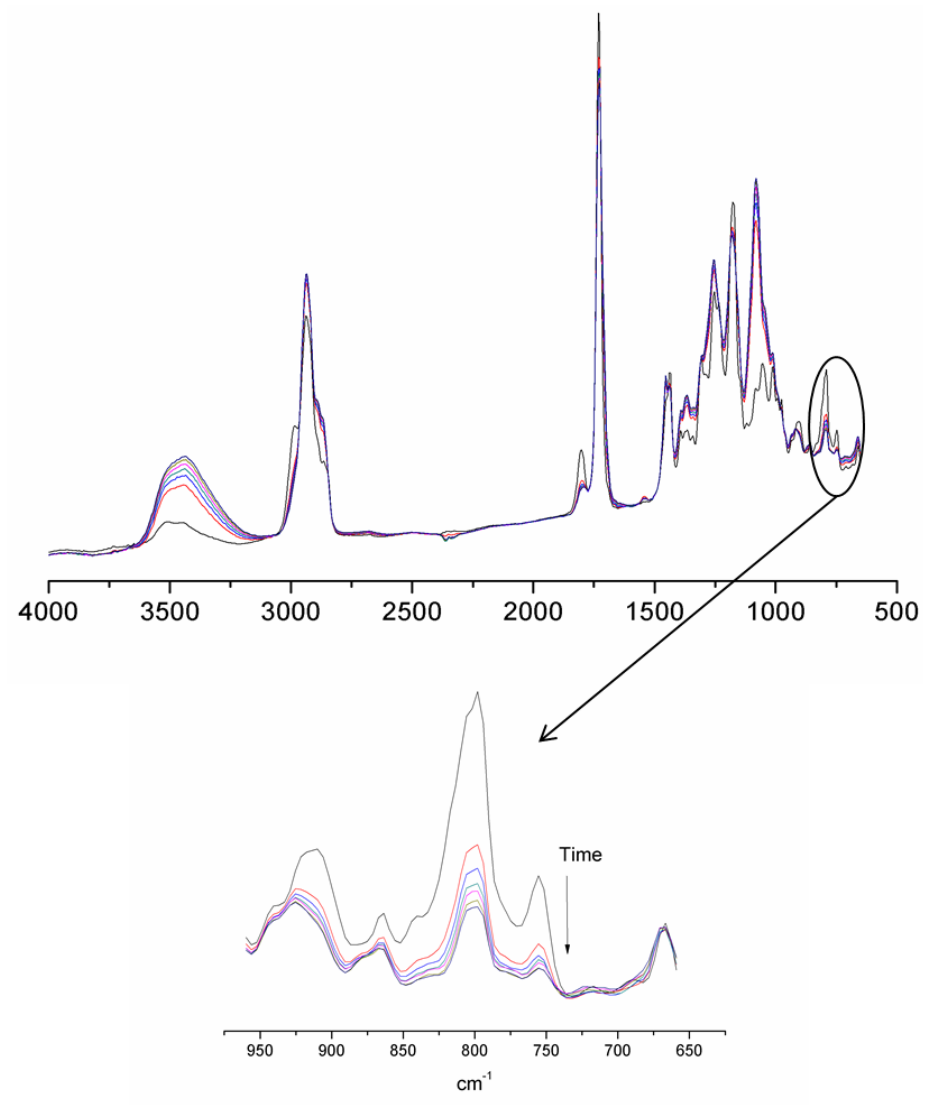

Figure 1: FTIR spectra with irradiation time for the pure epoxy resin over the entire region and a closed-up from 950 to $650 \mathrm{~cm}^{-1}$. Curing time $3 \mathrm{~min}$.

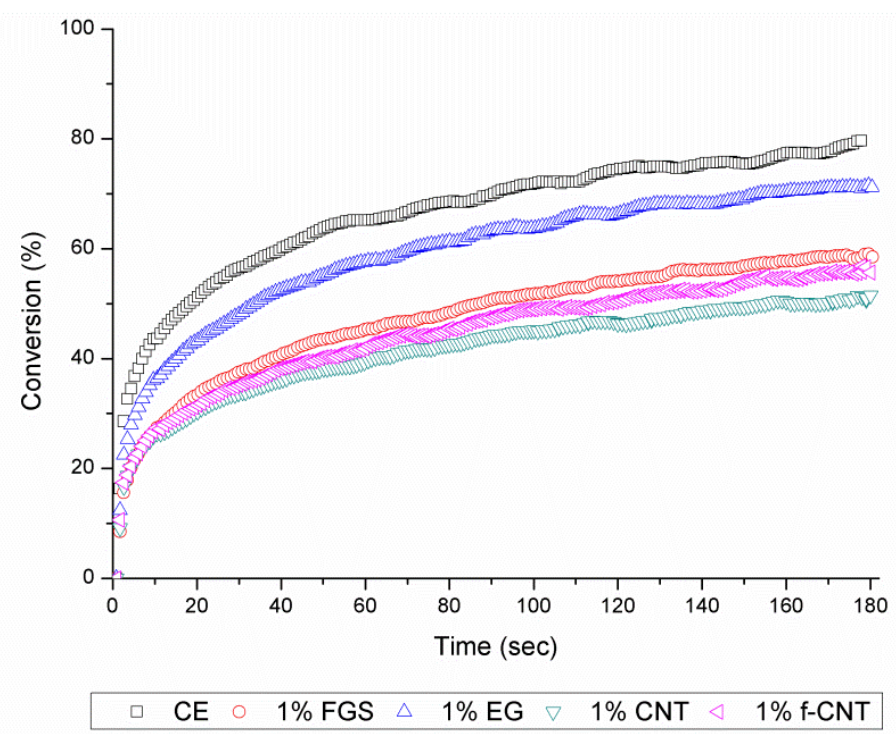

Figure 2: FTIR conversion curves for the neat resin and its nanocomposites at $1 w t . \%$ of nanofiller content. 
At the initial stages, the reactivity of the epoxy groups was very high for the pristine $\mathrm{CE}$ resin, and after 3 minutes of irradiation the final conversion of the epoxy band was around $80 \%$. This phenomenon was caused by the rise of $T_{g}$ of the system, due to the formation of a crosslinked polymer network hindering the mobility of active groups while the reaction progresses. We could observe that all carbon nanofillers decreased both the polymerization rate and the final conversion. Table 2 summarizes the value of the slopes at the initial stage and the final conversion values for the different photocured materials. The results suggested that the more staked structure of the graphite sheets in the EG led to higher reaction rates and a final conversion of $71 \%$ while the fillers at nanometer scale with higher specific surface area (SSA), FGS and MWCNT, had a greater absortion of the UV light leading to a stronger shielding effect with a final conversion between 50-60\%. No significant differences between MWCNT and FGS were observed.

Table 2: Conversion values and gel content of the nanocomposites with $1 \mathrm{wt} . \%$ loading fraction.

\begin{tabular}{|c|c|c|c|c|}
\hline & $\begin{array}{c}\text { Polymerization rate } \\
\left(\mathrm{sec}^{-1}\right)\end{array}$ & $\begin{array}{c}\text { Conversion }^{\mathbf{b}} \\
(\%)\end{array}$ & $\begin{array}{c}\text { Conversion }^{\mathrm{c}} \\
(\%)\end{array}$ & $\begin{array}{c}\text { Gel content } \\
(\%)\end{array}$ \\
\hline$\overline{C E}$ & 9.6 & 80 & 100 & 100 \\
\hline $1 \%$ FGS & 5.3 & 59 & 95 & 100 \\
\hline $1 \% \mathrm{EG}$ & 7.5 & 71 & 98 & 100 \\
\hline 1\% MWCNT & 5.5 & 51 & 98 & 99 \\
\hline $1 \%$ f-MWCNT & 5.6 & 56 & 88 & 100 \\
\hline
\end{tabular}

${ }^{a}$ Value of the slope at the initial stages of the conversion-time curves.

${ }^{b}$ Value of the plateau in the conversion curves with a UV-light intensity of $35 \mathrm{~mW} / \mathrm{cm}^{2}$ and an irradiation time of $3 \mathrm{~min}$. Film thickness of $25 \mu \mathrm{m}$.

${ }^{c}$ Determined by single spectra taken before and after 5 min of irradiation at $55 \mathrm{~mW} / \mathrm{cm}^{2}$. Film thickness of $25 \mu \mathrm{m}$. 
The observed slow down of the epoxy group conversion was due to the addition of a highly light-absorbing filler that reduces the generation of reactive species at the beginning of the photoinduced reaction. Hence, this competition in terms of light absorption between the nanofiller and the photoinitiator led to a less effective UVcuring process. Besides the shielding effect it can be expected that the rigid nanofillers reduce the mobility of the epoxy chains by increase of the viscosity of the photocurable formulation and as consequence the mobility of the reactive species is reduced decreasing the overall reactivity. Furthermore, it is known that the exothermal character of the cationic curing reaction of epoxy increases the temperature of the system and accelerates the crosslinking reaction. As the carboniuos nanofillers have a higher thermal conductivity than the resin, it is also expected that the heat generated by the curing reaction is being taken out of the matrix and therefore the reaction of the filled formulations is not accelerated as in the case of neat resin.

In order to overcome these effects, we demonstrated in our previous work [7] that the use of higher UV light intensities and longer irradiation times $\left(55 \mathrm{~mW} / \mathrm{cm}^{2}\right.$ and $7.5 \mathrm{~min}$ of irradiation time per side) was enough to develop a completely crosslinked polymer network with enhanced mechanical properties in FGS nanocomposites. In table 2, we also show the values of the conversion with these new processing conditions for $25 \mu \mathrm{m}$ thickness films and the gel content of the cured coatings evidencing the formation of a highly crosslinked network.

\section{Dielectric properties}

The dielectric properties of the photocured epoxy nanocomposites at different nanofiller contents measured at room temperature are depicted in Figure 3. The electrical conductivity and dielectric permittivity were significantly increased by the addition of 
all nanofillers in different ways. We can observe how the geometry of the filler played a dominant role in the formation of conductive pathways. The conductivity with just a small percentage $(0.1 \mathrm{wt} . \%)$ of non-functionalized rod-like filler became independent of the frequency in almost all the range of study. While the dielectric permittivity had the opposite behaviour, passing from being independent of the frequency to reveal a negative slope when the concentration was above the percolation threshold due to polarizarion effects between clusters inside the percolated system. This shift on the dielectric behavior is also attributed to the well-known Maxwell, Wagner and Sillars (MWS) effects in heterophase systems which basically consist on the polarization of the polymer-filler interface. The MWS interfacial polarization depends on several variables like: volume fraction of the conducting phase, the geometry of the filler, the dielectric permittivity of the matrix and the conductivity of the occluded phase [15]. These two drastic enhancements in conductivity and permittivity are evidences of the formation of an effective conductive MWCNT percolative network in the epoxy matrix. This was confirmed by the morphology investigation, as discussed below. On the other hand, when the resin was loaded with sheet-like nanofiller the increase of these two properties was much lower than for MWCNT and the thresholds appeared at higher concentrations, but in all cases the antistatic limit of $10^{-8} \mathrm{~S} / \mathrm{cm}$ is reached.

Extrapolating the broadband $\mathrm{AC}$ conductivity to $10^{-1} \mathrm{~Hz}$ it was possible to obtain $\mathrm{DC}$ conductivity values. Figure 4 shows DC conductivity for the nanocomposites studied as a function of nanofiller content. The filled areas correspond to the electrical percolation region for each system. We observed that the most effective filler in terms of the electrical percolation thershold was the MWCNT. The threshold values for the different fillers used agreed with those found in literature [16-18]. 

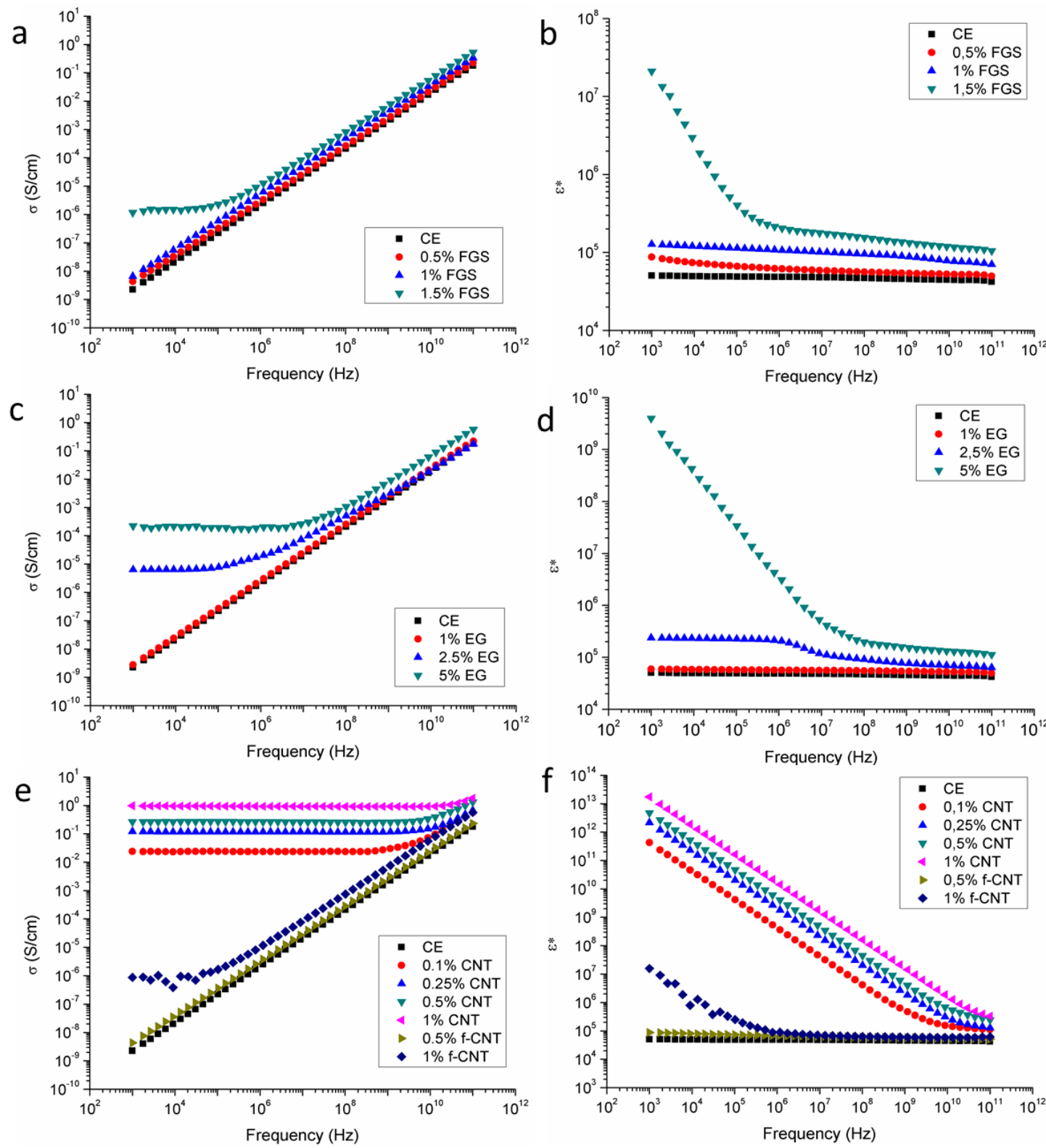

Figure 3: AC conductivity and permitivity versus frequency for all the fillers at different loading fractions. 


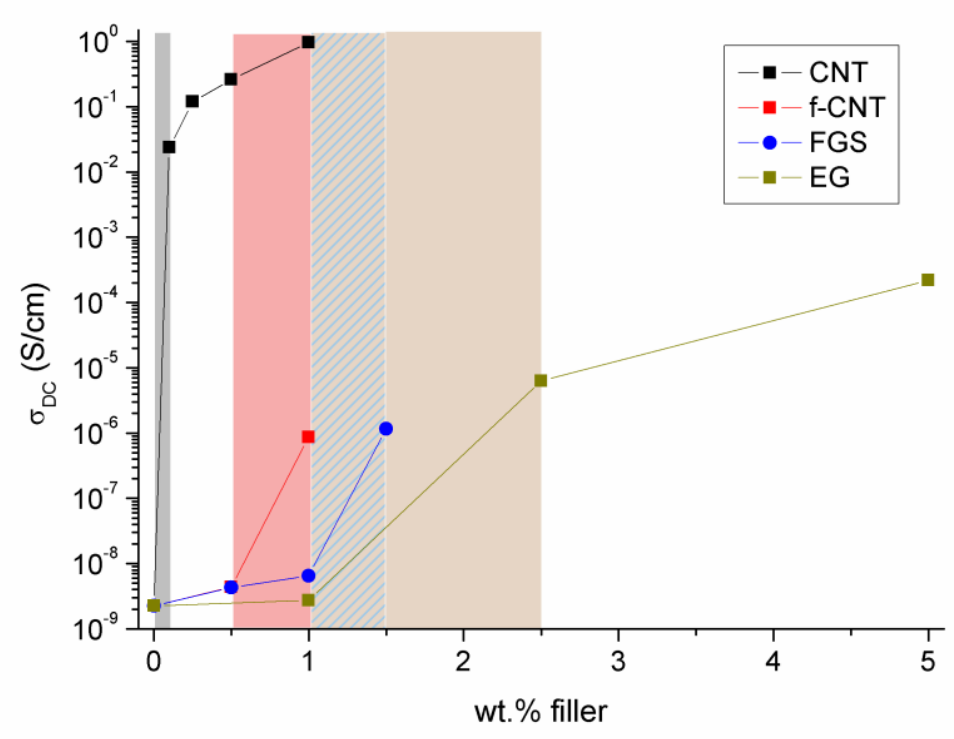

Figure 4: Values of the DC electrical conductivity of the epoxy nanocomposites as a function of the nanofiller content.

The differences in the percolation thresholds obtained were attributed to the geometry of the fillers. First of all, it would be easier for polymer chains to intercalate between MWCNT than between sheets facilitating the disentanglement of the MWCNT. Moreover sheet-like nanofiller would be more likely to form aggregates due to their inplane interactions and higher SSA. FGS have a wrinkled shape reducing the aspect ratio of the filler, in addition they are also able to roll themselves up into a tube-like structure with diameters larger than those of MWCNTs [19]. This was confirmed by morphological investigation, as discussed below: EG sheets were found forming big agglomerates leading to higher percolation thresholds. The rod-like geometry of the MWCNT could generate a huge number of overlapping contacts among them causing an effective electronic transport, while sheet-like nanofiller are connected through a shorter number of connections, such as plane-to-plane, edge-to-plane and edge-to-edge, 
which are not equally efficient from the electrical point of view [18]. The higher percolation threshold of f-MWCNTs compared to as-synthesized MWCNT was attributed to the disturbance of the crystalline graphitic structure of the MWCNT, reducing the number of $\pi$-electrons and generating more $\mathrm{sp}^{3}$-carbons which do not cause such as high electronic transport as $\pi$-electrons. Additionally, the reaction of the epoxy resin with the functional groups of the f-MWCNT could form an electrically insulating polymer layer around the nanotubes and, hence, hinder the electron tunneling between MWCNTs [20].

\section{Morphology}

We investigated the dispersion states of the different nanofillers in the epoxy resin by SEM and TEM inspections on cured materials, in order to get some conclusions of the morphology effect of the nanocomposites on their electric properties. We chose the highest loading fraction for each set of samples. Figures $5 \mathrm{a}$ and $\mathrm{b}$ are images at different magnifications of the nanocomposite with 1.5 wt.\% of FGS obtained by transmission electron microscopy. The achieved morphology showed a random and homogeneous distribution of single graphene sheet or stacks of a few nanometres adopting a wrinkled configuration.
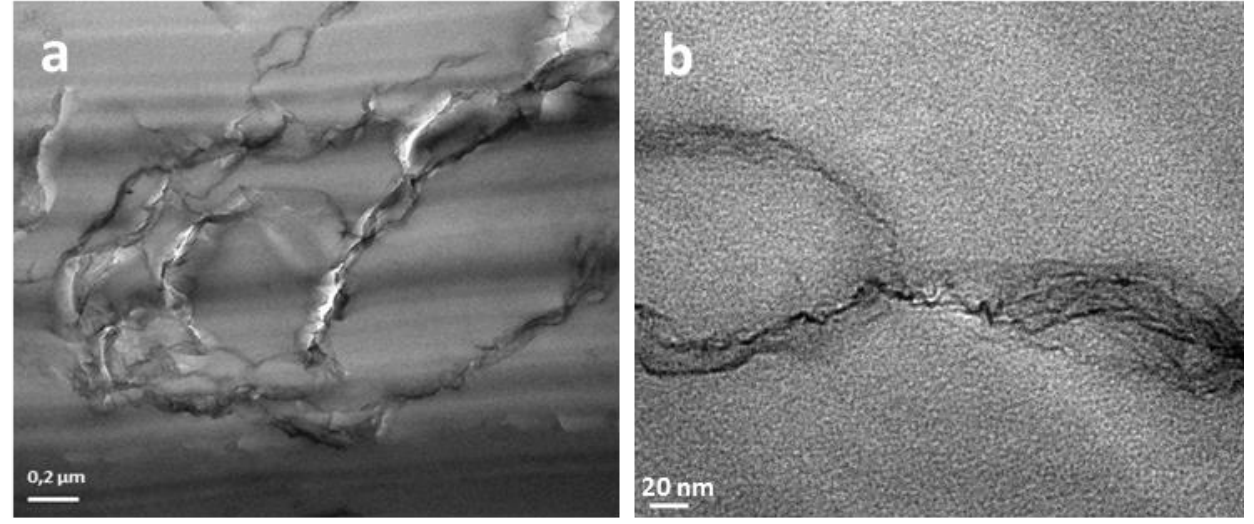

Figure 5: TEM images of the $1.5 \mathrm{wt} . \%$ FGS nanocomposite. 
Figure 6 contains two SEM images of the $5 \mathrm{wt} \%$ EG nanocomposite surface fracture where it is important to notice the scale of the filler. We observed agregates of several microns dispersed in the polymer matrix. The presence of these micrometer agglomerates made the formulation absorb less UV-light than the other fillers in the nanometer scale but also, as discussed above, the electrical conductivity was lower.
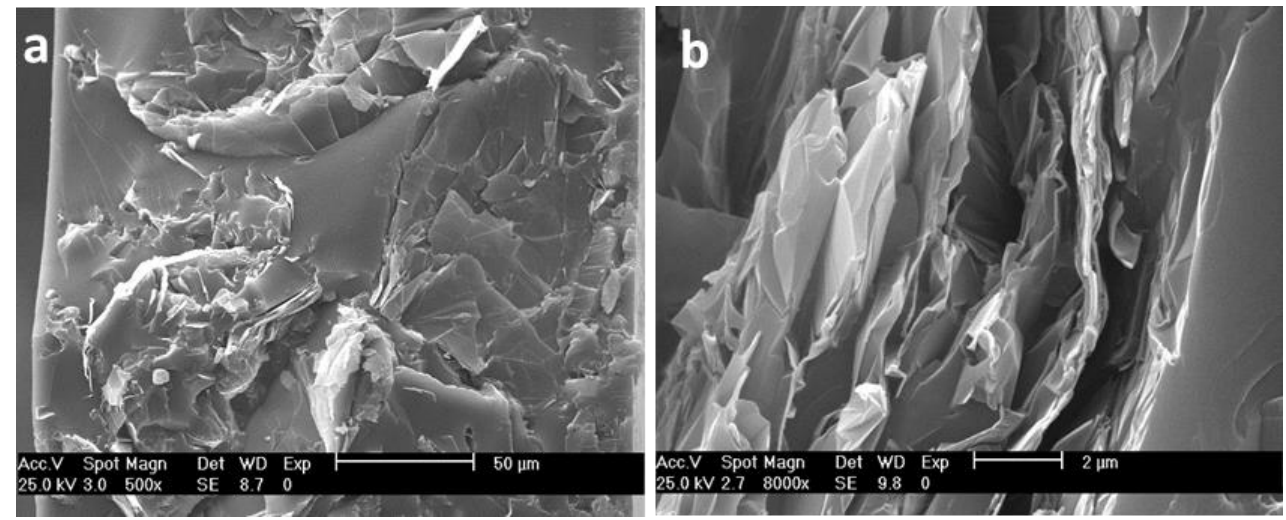

Figure 6: SEM images of the fracture surface of the 5 wt\% EG nanocomposite.

The SEM images shown in figure 7 correspond to the analysis of the cryo-fractured surface of the 1 wt.\% MWCNT and f-MWCNT nanocomposites. In both cases, the nanotubes were very well dispersed and spread out in the epoxy matrix without forming agglomerates.
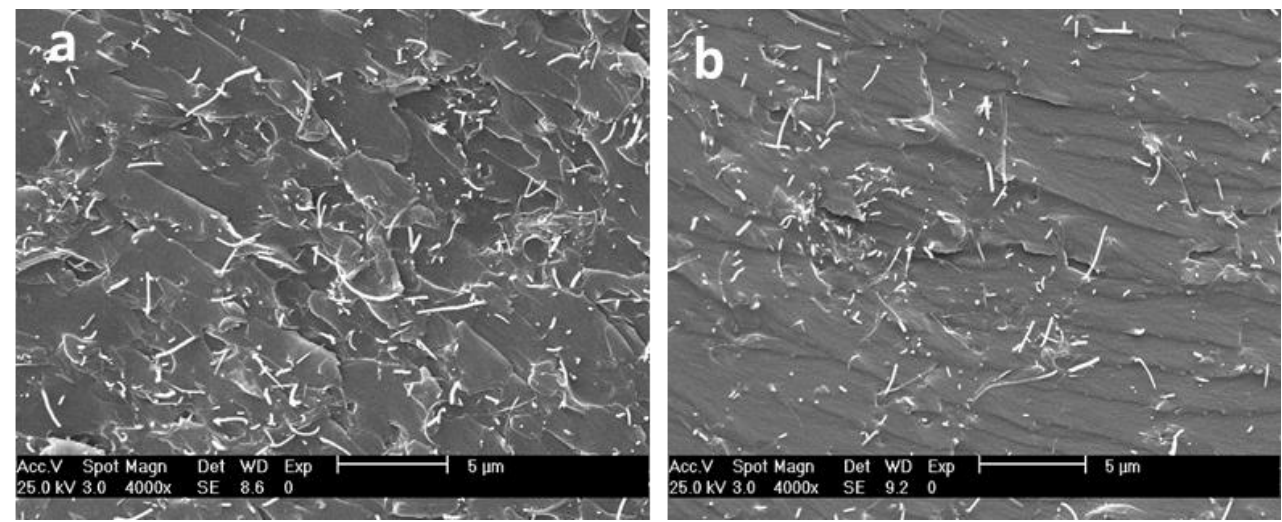

Figure 7: SEM images of the $1 \mathrm{wt} . \%$ a) MWCNT and b) $f$-MWCNT nanocomposites. 
It is important to mention that we found isolated MWCNT in the matrix while FGS with their sheet-like morphology formed stacked groups of a few wrinkle graphene sheets. This achieved dispersion morphology together with the geometry of the nanofillers influenced the final electrical properties of the nanocomposites, as previously discussed. Therefore the morphological investigations were strongly in agreement with the measured electrical properties.

In figure 8 , we show a TEM image of the $1 \mathrm{wt} \%$ MWCNT nanocomposite where it is possible to see the MWCNT network, responsible for the highly effective electronic transport of these nanocomposites.

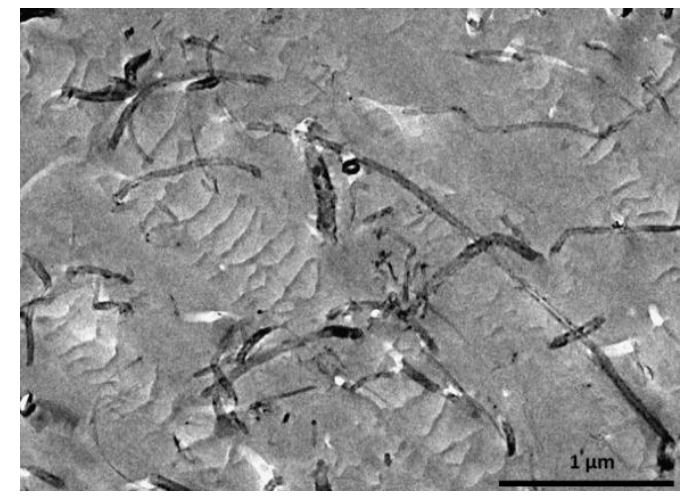

Figure 8: TEM image of the 1 wt.\% MWCNT nanocomposite with clear overlapping between MWCNTs.

\section{Dynamic mechanical analysis}

Viscoelastic characterization, glass transition temperature $\left(T_{g}\right.$ expressed as the maximum value of loss factor, $\tan \delta_{\max }$ ) and damping values, of the cured materials was carried out by DMTA analyses. Figure 9 shows the $\tan \delta$ curves as a function of temperature for the different prepared materials. It is possible to observe that the filled materials showed always higher $T_{g}$ values compared to the pristine cured epoxy matrix 
(Table 3), evidenced by the shift of the maximum of the $\tan \delta$ peaks, together with a decrease of the damping.

The DMTA results showed that the dynamic-mechanical properties of composites were greatly affected by the carboniuos nanofillers. It is well known that in particulate-filled polymers, the addition of rigid fillers can hinder the polymer chain movements leading to a damping decrement and a shift of $T_{g}$ values to higher temperatures. In this study a significant reduction of damping (here evaluated as $\tan \delta \max$ ) was observed in the nanocomposites, together with a strong increment in $T_{g}$ values.

These changes in both $T_{g}$ and damping are generally attributed to a strong filler-matrix interface; Thus, nanofillers with functional groups on their surface (FGS and fMWCNT) evidenced higher damping effect because of the covalent bonding between the filler and the polymer chains. The largest enhancement in the $T_{g}$ was obtained with FGS (20 ${ }^{\circ} \mathrm{C}$ with 1 wt.\%) due to the formation of a large and chemically bonded interface at the proximity of the filler. FGS nanocomposite behaved even better than the f-MWCNT and MWCNT from the dynamic mechanical point of view. Raffie et. al. [21] already concluded that graphene systems outperform the mechanical properties of MWCNT nanocomposites because of the more efficient adhesion to the matrix and higher SSA of the graphene sheets. 


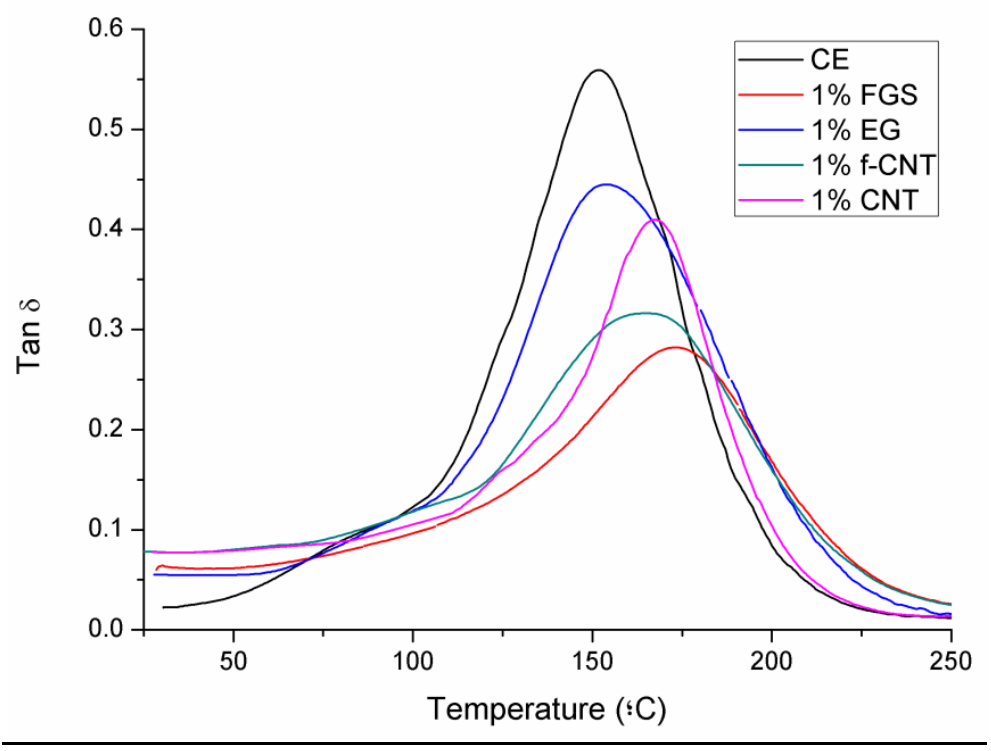

Figure 9: Tan $\delta$ curves obtained by DMTA analysis for the different nanocomposites at 1 wt.\% loading fraction.

Even though the E' (Figure 10) of the nanocomposites at low temperature is lower than the $E^{\prime}$ of the neat resin, we can observe an important hindering effect of the nanofiller on the polymer chain mobility extending the performance of the elastic modulus to higher temperatures above the $\mathrm{T}_{\mathrm{g}}$.

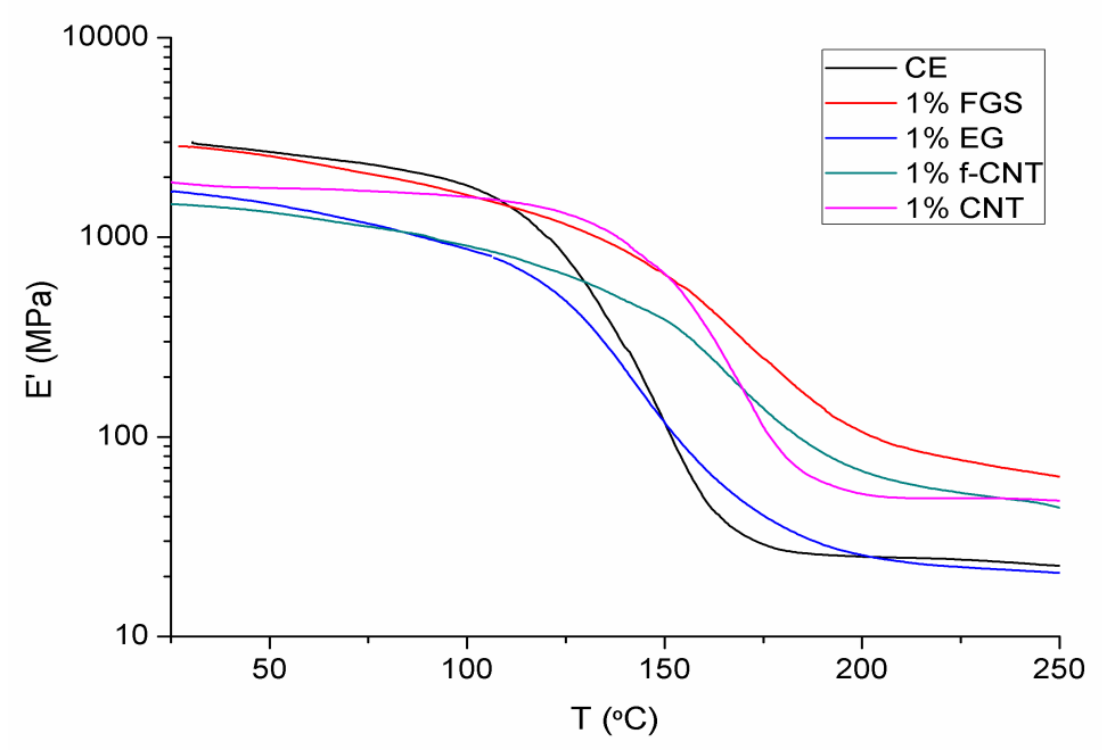


Figure 10: E' curves obtained by DMTA analysis for the different nanocomposites at 1 wt.\% loading fraction.

Table 3: $T_{g}$ values of the cured nanocomposites.

\begin{tabular}{cccccc}
\hline Sample & CE & 1\% FGS & 1\% EG & 1\% f-MWCNT & 1\% MWCNT \\
\hline$T_{g}\left({ }^{\circ} \mathrm{C}\right)$ & 152 & 173 & 154 & 165 & 167 \\
\hline
\end{tabular}

\section{Surface hardness}

The good dynamic mechanical behaviour of the nanocomposites can be accompanied by an enhancement of the surface hardness. The results of microindentation tests performed on the surface of the photocured samples are shown in Figure 11. Surface hardness values (Figure 11.a) showed that the un-filled epoxy resin and its nanocomposites had very similar responses. Although, a slight increase in the indentation hardness (Hit) could be observed particularly for the sheet-like nanofillers. Again, the better mechanical performance of the sheet-like nanofillers was attributed to the enhanced specific area and geometry of the FGS and/or to a better mechanical interlocking of the nanofiller/matrix interface [21]. We also plotted in Figure 11.b the loadingmaintenance-unloading process of the microindentation test. In these plots, the differences in the mechanical response between samples were more evident. At the same load the indentation depth for the nanocomposites was lower than for the pristine epoxy resin evidencing the reinforcing effect of the nanofillers. 

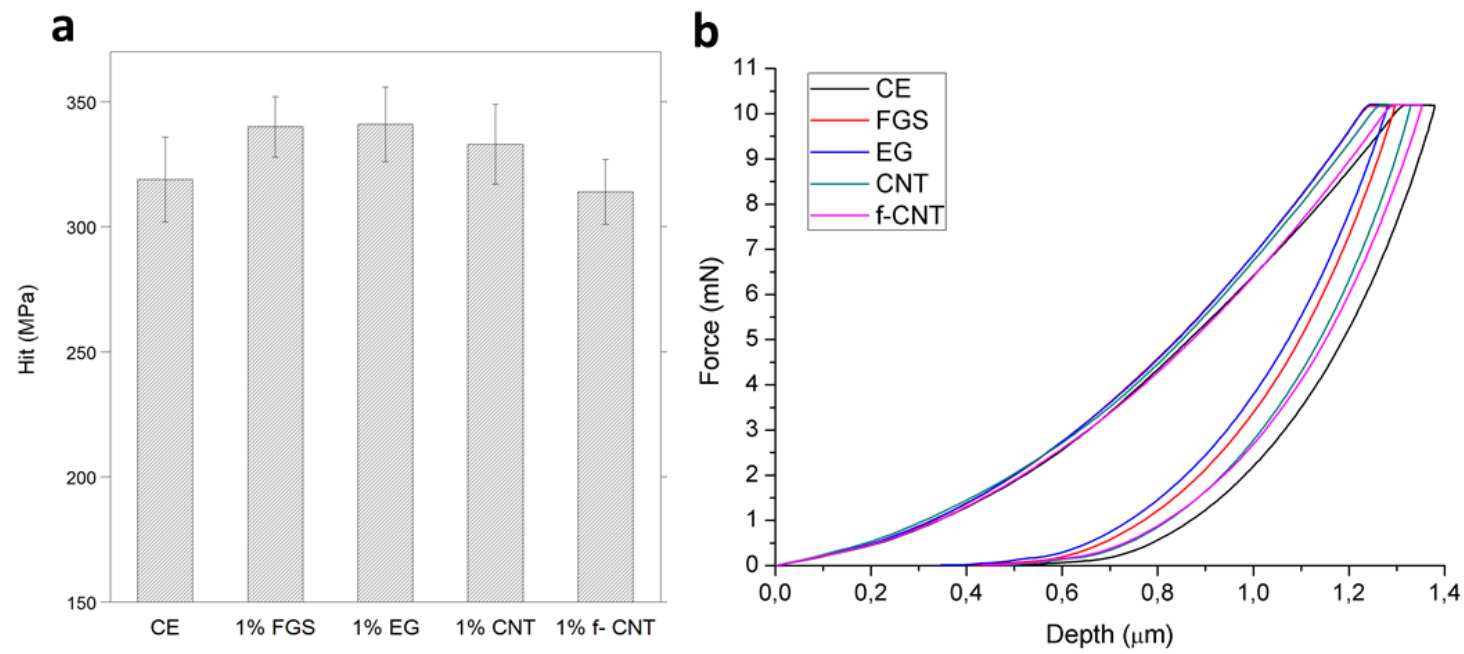

Figure 11: (a) Surface hardness values and (b) load vs depth curves.

\section{CONCLUSIONS}

Three different types of carbon based nanofillers, i.e. MWCNT, FGS and EG, were introduced into a photo-curable epoxy resin formulation. The extent of reaction was slightly delayed by UV-light due to both a shielding effect, an increase of the viscosity of the filled-formulation, which decreased the mobility of the reactive species. This phenomenon was more pronounced with nanometric fillers with higher aspect ratio and SSA (MWCNT and FGS). Adjusting the process conditions, we were able to develop nanocomposites characterized by high final conversion and gel values.

By analyzing the dielectric properties of the cured nanocomposites we found different percolation thresholds depending on the type of filler. The highest enhancement in electrical properties was achieved in the MWCNT composites while the sheet-like based nanocomposites were lower. These differences were attributed to the geometry of the filler, the filler dispersion and the more effective MWCNT network to the electronic transport through overlapping of nanotubes. Nevertheless, all types of nanocomposites evaluated shifted the insulating behavior of the pristine epoxy resin to an antistatic 
material. However, FGS was the most effecting filler for epoxy resin which showed an increase in the $T_{g}$ of more than $20^{\circ} \mathrm{C}$ at 1 wt. $\%$.

\section{ACKNOWLEDGMENTS}

The work was supported by the Spanish Ministry of Science and Innovation (MICINN) under project MAT 2010-18749. MMG thanks the CSIC for a JAE-Pre grant.

\section{REFERENCES}

1. Leng J and Lau AKT. Multifunctional polymer nanocomposites. Boca Raton: CRC Press, 2011.

2. Verdejo R, Bernal MM, Romasanta L, and Lopez Manchado M. Journal of materials chemistry 2011;21(10):3301-3310.

3. Chou T-W, Gao L, Thostenson E, Zhang Z, and Byun J-H. Composites science and technology 2010;70(1):1-19.

4. Enoki T, Endo M, and Suzuki M. Graphite intercalation compounds and applications: Oxford University Press, 2003.

5. Potts J, Dreyer D, Bielawski C, and Ruoff R. Polymer 2011;52(1):5-25.

6. Sangermano M, Pegel S, Pötschke P, and Voit B. Macromolecular Rapid Communications 2008;29(5):396-400.

7. Martin-Gallego M, Verdejo R, Lopez-Manchado MA, and Sangermano M. Polymer 2011;52(21):4664-4669.

8. Fouassier JP and Rabek JF. Radiation Curing in Polymer Science and Technology. London: Chapman and Hall, 1993. 
9. Sangermano M, Buongiovanni R, Malucelli G, and Priola A. "New Developments in Cationic Photopolymerization: Process and Properties". New York: Nova Science Publisher Inc, 2006.

10. Verdejo R, Lamoriniere S, Cottam B, Bismarck A, and Shaffer M. Chemical communications (London. 1996) 2007(5):513-515.

11. Verdejo R, Staempfli R, Alvarez Lainez M, Mourad S, and Rodriguez-Perez MA. Composites science and technology 2009;69(10):1564-1569.

12. Schniepp HC, Li JL, McAllister MJ, Sai H, and Herrera Alonso M. The journal of physical chemistry. B 2006;110(17):8535-8539.

13. Verdejo R, Barroso Bujans F, Rodriguez Perez M, de Saja J, and Lopez Manchado M. Journal of materials chemistry 2008;18(19):2221-2226.

14. Poetschke P, Abdel Goad M, Pegel S, Jehnichen D, and Mark J. Journal of macromolecular science. Pure and applied chemistry 2010;47(1):12-19.

15. Hayward D, Pethrick RA, and Siriwittayakorn T. Macromolecules 1992;25(5):1480-1486.

16. Chen G-H, Wu D-J, Weng W-G, and Yan W-L. Journal of Applied Polymer Science $2001 ; 82(10): 2506-2513$.

17. Bauhofer $\mathrm{W}$ and Kovacs J. Composites science and technology 2009;69(10):1486-1498.

18. Du J, Zhao L, Zeng Y, Zhang L, and Li F. Carbon 2011;49(4):1094-1100.

19. Viculis LM, Mack JJ, and Kaner RB. Science 2003;299(5611):1361-1361.

20. Gojny FH, Wichmann MHG, Fiedler B, Kinloch IA, and Bauhofer W. Polymer 2006;47(6):2036-2045.

21. Rafiee M, Rafiee J, Wang Z, Song H, and Yu Z-Z. ACS nano 2009;3(12):38843890. 


\section{FIGURE CAPTIONS}

Figure 1: FTIR spectra with irradiation time for the pure epoxy resin over the entire region and a closed-up from 950 and $650 \mathrm{~cm}^{-1}$. Curing time $3 \mathrm{~min}$.

Figure 2: FTIR conversion curves for the neat resin and its nanocomposites at $1 \mathrm{wt} . \%$ of nanofiller content.

Figure 3: TEM images of the $1.5 \mathrm{wt} . \%$ FGS nanocomposite.

Figure 4: SEM images of the fracture surface of the $5 \mathrm{wt} \%$ EG nanocomposite.

Figure 5: SEM images of the $1 \mathrm{wt} . \%$ a) MWCNT and b) f-MWCNT nanocomposites.

Figure 6: TEM image of the $1 \mathrm{wt} . \%$ MWCNT nanocomposite with clear overlapping between MWCNTs.

Figure 7: AC conductivity and permitivity versus frequency for all the fillers at different loading fractions.

Figure 8: Values of the DC electrical conductivity of the epoxy nanocomposites as a function of the nanofiller content.

Figure 9: Tan $\delta$ curves obtained by DMTA analysis for the different nanocomposites at 1 wt.\% loading fraction.

Figure 10: (a) Surface hardness values and (b) load vs depth curves. 


\section{CATIONIC PHOTOCURED EPOXY NANOCOMPOSITES}

FILLED WITH DIFFERENT CARBON FILLERS

M. Martin-Gallego ${ }^{1 *}$, M. Hernández ${ }^{1}$, V. Lorenzo ${ }^{2}$, R. Verdejo ${ }^{1}$, M.A. LopezManchado $^{1}$, M. Sangermano ${ }^{3 *}$

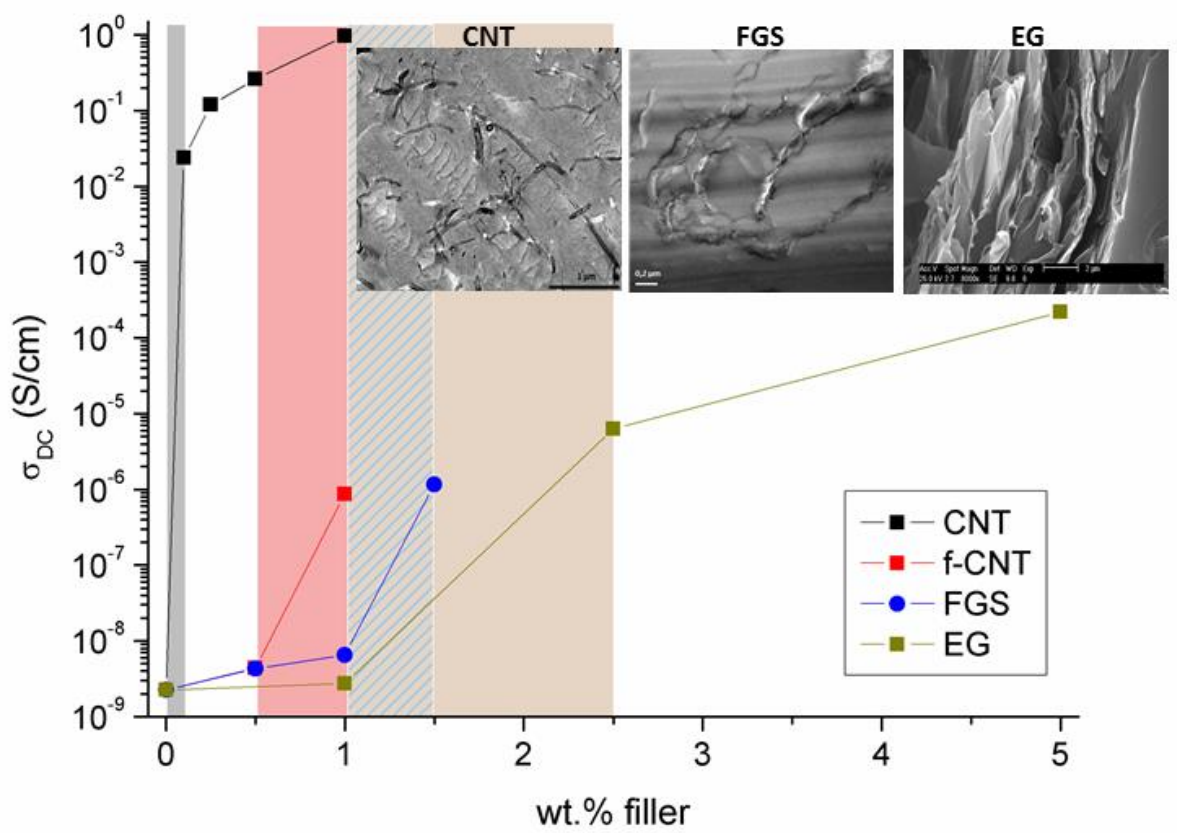

